\title{
HMGB1 promotes the secretion of multiple cytokines and potentiates the osteogenic differentiation of mesenchymal stem cells through the Ras/MAPK signaling pathway
}

\author{
LIN FENG $^{1}$, DETING XUE $^{2}$, ERMAN CHEN $^{2}$, WEI ZHANG ${ }^{2}$, XIANG GAO $^{2}$, \\ JIAWEI YU ${ }^{2}$, YADONG FENG ${ }^{2}$ and ZHIJUN PAN ${ }^{2}$ \\ ${ }^{1}$ Department of Orthopedics, The First People's Hospital of Xiaoshan, Hangzhou, Zhejiang 311200; \\ ${ }^{2}$ Department of Orthopedics, Second Affiliated Hospital, School of Medicine, \\ Zhejiang University, Hangzhou, Zhejiang 310009, P.R. China
}

Received March 10, 2015; Accepted April 6, 2016

DOI: $10.3892 /$ etm.2016.3857

\begin{abstract}
High mobility group box 1 (HMGB1) protein has been previously been detected in the inflammatory microenvironment of bone fractures. It is well known that HMGB1 acts as a chemoattractant to mesenchymal stem cells (MSCs). In the present study, the effects of HMGB1 on cytokine secretion from MSCs were determined, and the molecular mechanisms underlying these effects of HMGB1 on osteogenic differentiation were elucidated. To detect cytokine secretion, antibody array assays were performed, which demonstrated that HGMB1 induced the differential secretion of cytokines that are predominantly associated with cell development, regulation of growth and cell migration, stress responses, and immune system functions. Moreover, the secretion of epidermal growth factor receptor (EGFR) was significantly upregulated by HMGB1. The EGFR-activated Ras/MAPK pathway regulates the osteogenic differentiation of MSCs. These results suggested that HMGB1 enhances the secretion of various cytokines by MSCs and promotes osteogenic differentiation via the Ras/MAPK signaling pathway. The present study may provide a theoretical basis for the development of novel techniques for the treatment of bone fractures in the future.
\end{abstract}

\section{Introduction}

High mobility group box 1 (HMGB1) protein is a highly conserved DNA-binding protein that was named according to its high gel mobility during electrophoresis $(1,2)$. HMGB1 is a highly conserved DNA-binding protein that mediates cytokine

Correspondence to: Miss. Zhijun Pan, Department of Orthopedics, Second Affiliated Hospital, School of Medicine, Zhejiang University, 88 Jiefang Road, Hangzhou, Zhejiang 310009, P.R. China E-mail: zepzj@163.com

Key words: high mobility group box 1, cytokines, osteoblastic differentiation, Ras/MAPK signal pathway effects and, as a result, modulates gene transcription, stabilizes nucleosomes, promotes inflammatory responses, and regulates neural growth and tumor metastasis (3-6). In bone fractures, HMGB1 was found to be released into the extracellular environment by active secretion from stimulated cells as well as by passive discharge from necrotic cells (7).

Mesenchymal stem cells (MSC) are multipotent stromal cells that can be induced to differentiate into various types of mesenchymal tissues in specific in vivo environments. MSCs possess several stem cell characteristics, including self-renewal, pluripotency and homing, therefore they are considered the predominant source of stem cells for fracture restoration. Migration and osteogenic differentiation of MSCs has a critical role during the partial coalescence of fractures (8-10). It is also widely known that MSCs are capable of secreting various types of cytokines, including stem cell factor (SCF), thrombopoietin and interleukin-6 $(11,12)$. These cytokines regulate stem cell differentiation, direct migration and mediate inflammatory processes (13-15). Therefore, cytokines secreted from MSCs may affect fracture coalescence. However, in an inflamed environment, such as during partial coalescence of fractures, the stimulation of inflammatory factors may alter the concentration and type of cytokines secreted from MSCs (16). This phenomenon may directly affect various cytokine-dependent biological processes and subsequently modify the characteristics and functions of MSCs and related cells. HMGB1 is ubiquitously present in the inflamed microenvironment of fractures and is considered to be a pro-inflammatory cytokine $(17,18)$. Therefore, we hypothesized that, consistent with other inflammatory factors, HMGB1 may affect cytokine secretion from MSCs.

In the present study, antibody array assays were performed to detect cytokine secretion from MSCs upon HMGB1 stimulation. As certain cytokines were differentially secreted from MSCs upon the treatment with HMGB1, the roles of these cytokines were analyzed in an attempt to elucidate the overall effects of HMGB1 on the biological functions of MSCs. Although the promoting actions of HMGB1 on the MSC osteogenic differentiation have previously been reported (19), the detailed mechanisms of these effects are yet to be investigated and elucidated. Therefore, the aim of the present study was to 
elucidate the mechanisms underlying the effects of HMGB1 on MSCs using antibody array analysis. These results may provide a basis for developing novel approaches in bone fracture-healing therapy.

\section{Materials and methods}

Reagents. MSCs and basal culture medium were purchased from Cyagen Biosciences, Inc., (Santa Clara, CA, USA). Recombinant human HMGB1 protein and fetal bovine serum were purchased from Sigma-Aldrich (St. Louis, MO, USA). Ras inhibitor (Selleckchem, Houston, TX, USA), which was a transferase inhibitor for H-Ras and K-Ras, was used at a concentration of $5 \mu \mathrm{M}$.

Isolation and culture-expansion of human bone marrow MSCs. Adherent MSCs were trypsinized and passaged once cell confluence reached $\sim 80 \%$. Cells at passage 3-5 were used in the present experiments.

Assays for osteogenic differentiation. To induce osteogenic differentiation, MSCs were cultured in basal culture medium supplemented with fetal bovine serum (FBS).

Total RNA extraction and reverse transcriptase-quantitative polymerase chain reaction ( $R T-q P C R)$. To observe MSC differentiation following exposure to HMGB1, MSCs were cultured in basal culture medium or $25 \mathrm{ng} / \mathrm{ml} \mathrm{HMGB}-1$ for 5 days. Total RNA was extracted with TRIzol reagent (Thermo Fisher Scientific, Inc., Waltham, MA, USA) according to the manufacturer's protocol. RT-qPCR was performed to observe the expression of osteoblastic markers using a StepOne Plus Real-Time PCR system with SYBR Green (Roche Diagnostics, Basel, Switzerland) as a double-strand DNA-specific binding dye. Primer sequences were as follows: Osteocalcin $(\mathrm{OCN})$, forward 5'-AAG CAGGAGGGCAATAAGGT and reverse, CAAGCAGGGTTA AGCTCACA; and GAPDH, forward 5'-CGTCCCGTAGAC AAAATGGT and reverse 5'-GGCTGGTGGTCCAGGGGTCT (Sangon Biotech Co., Ltd., Shanghai, China). According to the manufacturer's protocol, DNA hydrolase was used to remove genomic DNA. The reaction mixture included buffer $(5 \mu \mathrm{l})$, dNTP $(4 \mu 1)$, primer $(4 \mu 1)$, Taq $(1 \mu \mathrm{l})$, sample $(1 \mu \mathrm{l}), \mathrm{SYBR}$ Green $(1 \mu \mathrm{l})$ and $\mathrm{ddH}_{2} \mathrm{O}$ to make a total volume of $50 \mu \mathrm{l}$. Thermal cycling conditions were as follows: $95^{\circ} \mathrm{C}$ for $30 \mathrm{sec}$ and 40 cycles of $95^{\circ} \mathrm{C}$ for $5 \mathrm{sec}$ and $60^{\circ} \mathrm{C}$ for $35 \mathrm{sec}$. Relative target gene expression levels were calculated according to the $2^{-\Delta \Delta \mathrm{Cq}}$ method (20). All PCR reactions were performed in triplicate. mRNA quantification of the target genes and the GAPDH housekeeping gene was performed in separate tubes.

Alkaline phosphatase (ALP) staining and activity assay. MSCs $\left(10^{4}\right.$ cells $\left./ \mathrm{cm}^{2}\right)$ were seeded into 24 -well plates and cultured in basal culture medium. After one week, ALP activity was assessed using a BCIP/NBT ALP color development kit (Beyotime Institute of Biotechnology, Haimen, China), according to the manufacturer's protocol. For the quantitative determination of ALP activity, the MSCs were incubated with p-nitrophenol phosphate (Beyotime Institute of Biotechnology) as a substrate, washed with PBS buffer and lysed with $0.1 \%$ Triton X-100 in $10 \mathrm{mM}$ Tris HCL, (pH 9.0). Absorbance was determined at $405 \mathrm{~nm}$ using a microplate reader and compared with p-nitrophenol standard titration curve. Data were presented as the mean \pm standard deviation $(n=3)$.

Western blotting. Cells were harvested and lysed in RIPA buffer (Cyagen Biosciences, Inc.) supplemented with protease inhibitors. Following concentration determination $(5.534 \mu \mathrm{g} / \mu \mathrm{l})$, protein samples $(60 \mu \mathrm{g})$ were separated by $10 \%$ sodium dodecyl sulfate-polyacrylamide gel electrophoresis and transferred to a polyvinylidene difluoride membrane by western blotting. Membranes were blocked in 5\% skim milk for $1 \mathrm{~h}$ and incubated with antibodies against GTP-Ras (cat. no. 16117; 1:500; Active Ras Detection kit; Thermo Fisher Scientific, Inc.), Ras (cat. no. ab52939; Abcam, Cambridge, UK), extracellular-signal-regulated kinases (ERK; cat. no. ab196883; 1:500; Abcam), phosphorylated (p-)ERK (cat. no. ab65142; 1:500; Abcam), p38 (cat. no. 9212; 1:1,000; Abcam), p-p38 (cat. no. 9215; 1:1,000; Abcam) and $\beta$-actin (cat. no. dc-130301; 1:2,000; Santa Cruz Biotechnology, Inc., Dallas, TX, USA), respectively, at $4^{\circ} \mathrm{C}$ overnight. Following incubation with horseradish peroxidase-conjugated goat anti-rabbit secondary antibodies (cat. no. 31210; 1:5,000; Thermo Fisher Scientific, Inc.), immunoreactive proteins were visualized using enhanced chemiluminescence reagent (Thermo Fisher Scientific, Inc.). Relative quantification of the bands was performed using ImageJ software (version 5.0; National Institutes of Health, Bethesda, MA, USA).

Antibody arrays. Soluble proteins in the medium of the stromal cell lines were measured using the Human Cytokine Array G1000 (AAH-CYT-G1000; RayBiotech Inc., Norcross, GA, USA), according to the manufacturer's protocol. These arrays can detect 120 proteins, respectively. Stromal cells were plated n Dulbecco's modified Eagle medium (DMEM) supplemented with $10 \%$ FBS 3 days prior to the experiment and were $75-90 \%$ confluent when the media were collected and filtered. DMEM containing 10\% FBS was also hybridized to the arrays and used for normalization. Ten technical and biological replicates were performed, and both exhibited a high correlation (correlation coefficient, $>0.9$ ) (data not shown). Hybridization was performed overnight at $4^{\circ} \mathrm{C}$. All slides were scanned using a Gene Pix 4000B Microarray Scanner (Molecular Devices, LLC, Sunnyvale, CA, USA) and analyzed using Gene Pix Pro 6.0 software. The median signal score was averaged across the triplicates on each array. Results were subsequently normalized using internal controls by subtracting the values for the control DMEM supplemented with 10\% FBS.

Statistical analysis. Statistical significance was determined using the two-tailed Student's t-test, assuming equal variances. The $\chi^{2}$ test was used to compare rates. SPSS version 17.0 (SPSS, Inc., Chicago, IL, USA) was used to analyze the data. $\mathrm{P}<0.05$ was considered to indicate a statistically significant difference.

\section{Results}

Effects of HMGB1 on cytokine secretion from MSCs. To determine the influence of HMGB1 stimulation on human MSCs at the protein level, antibody arrays were performed. The same three groups were stimulated with $25 \mathrm{ng} / \mathrm{ml}$ HMGB1 and compared with non-stimulated cultures (Fig. 1A). In line 
A

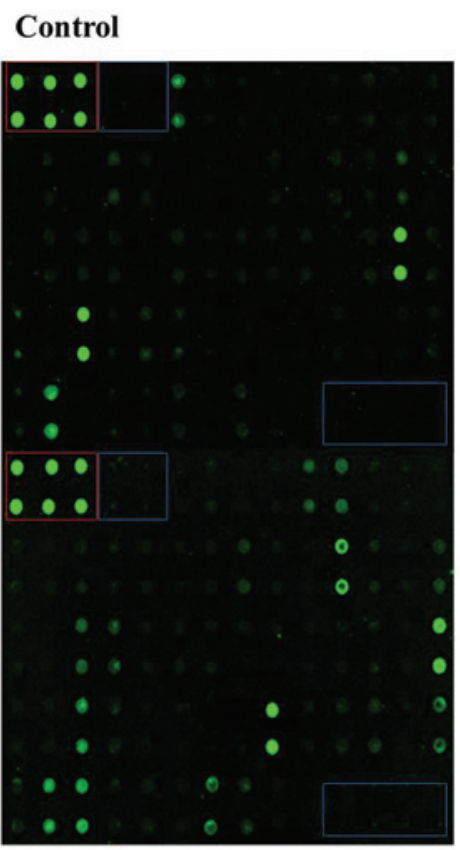

Treatment

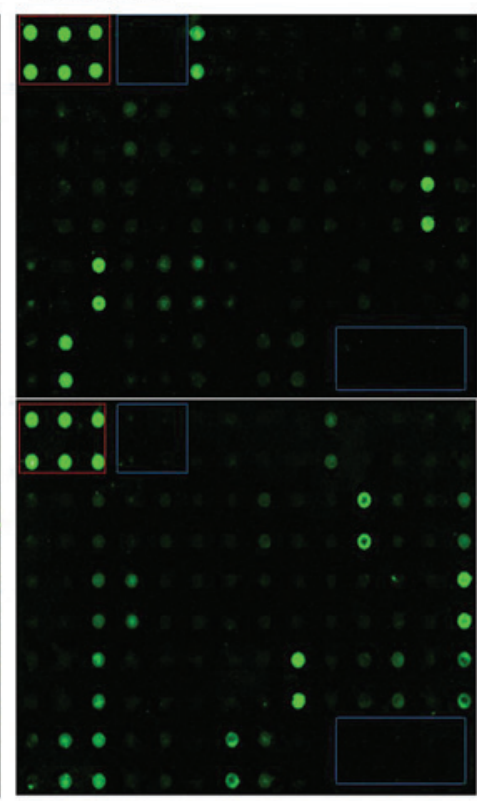

B

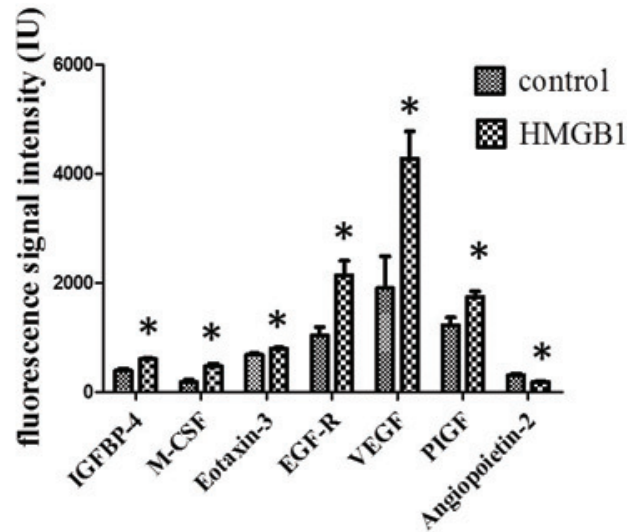

C

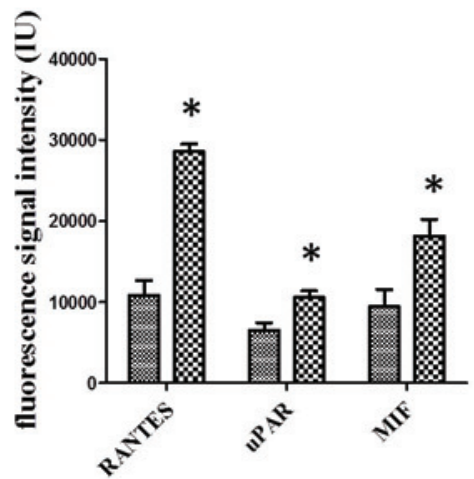

Figure 1. HMGB1-induced cytokine secretion. (A) Secretion of cytokines in cell culture supernatant was measured using antibody array analysis. The blue box indicates the negative controls, whereas the red box denotes the positive controls. Spots were measured densitometrically. Following subtraction of the negative control and normalization to the positive control, the spots of stimulated MSCs were compared to the corresponding spots of the unstimulated MSCs. (B and C) Quantification of antibody array results for cytokines with increased secretion following HMGB1 stimulation. * $<<0.05$ vs. the control. IGFBP, insulin-like growth factor binding protein; M-CSF, macrophage colony stimulating factor; EGFR, epidermal growth factor receptor; VEGF, vascular endothelial growth factor; PIGF, placental growth factor; CCL-5, chemokine C-C motif ligand 5; uPAR, urokinase plasminogen activator receptor; MIF, macrophage migration inhibitory factor; HMGB1, high mobility group box 1 .

with the search criteria for differential secretion, which were $>1.5$-fold induction or repression and $\mathrm{P}<0.05$, bioinformatic data analysis detected 10 cytokines which were differentially secreted between the groups. Increased cytokine secretion was detected for insulin-like growth factor binding protein 4, macrophage colony stimulating factor (M-CSF), eotaxin-3, epidermal growth factor receptor (EGFR), vascular endothelial growth factor (VEGF), placental growth factor, angiopoetin-2, chemokine $\mathrm{C}-\mathrm{C}$ motif ligand 5 (CCL-5), urokinase plasminogen activator receptor and macrophage migration inhibitory factor following induction with HMGB1 (Figs. 1B and C). These results suggest that $\mathrm{HMGB} 1$ promotes the secretion of multiple cytokines from MSCs.

Functions of the differentially secreted cytokines from MSCs. Hierarchical clustering of these 10 cytokines was performed with normalized cytokines secretion values for the two distinct groups: the HMGB1-treated group and the non-stimulated control group (Fig. 2). Six differentially secreted cytokines were visualized as induced, as indicated by red, and four cytokines were repressed, as indicated by green.

Notably, among the six induced cytokines, three cytokines, EGFR, M-CSF and VEGF, were associated with Ras signaling, and three cytokines were involved in signal transduction: EGF-R, CCL-5 and VEGF. These 10 differentially secreted cytokines have also been associated with cell development (5), the regulation of growth (5) and cell migration (8), response to stress (12) and immune system processes (9) according to the Gene Ontology database (http://amigo.geneontology.org/amigo) for annotation, visualization and integrated discovery. Various differentially secreted cytokines were associated with more than one biological process.

Some of the differentially secreted cytokines detected in the present study also have a role in other molecular and cellular functions, such as cellular growth (such as M-CSF) and proliferation (such as macrophage migration inhibitory factor), cell morphology (such as RANTES) and cellular development (such as placental growth factor). Based on the 10 differentially secreted cytokines, the cytokine-cytokine receptor interaction pathway (0460hsa in KEGG) was demonstrated to be the dominant pathway that was influenced by HMGB1. Here, the secretion of CCL5, CCL26, VEGFA and CSF1 was induced (Fig. 3). The results demonstrate that the differentially secreted cytokines serve an important role in the biological function of MSCs.

HMGB1-treated MSCs activate Ras/MAPK pathway to promote MSC osteogenic differentiation. Antibody array assay analysis demonstrated that the expression of EGFR was markedly increased in the MSCs following stimulation with HMGB1. It has previously been reported that the interaction between EGF and EGFR activates Ras/MAPK signaling pathway (21). To investigate whether Ras/MAPK signaling pathway was activated in MSCs, the protein levels of GTP-Ras, p-p38 and p-ERK 


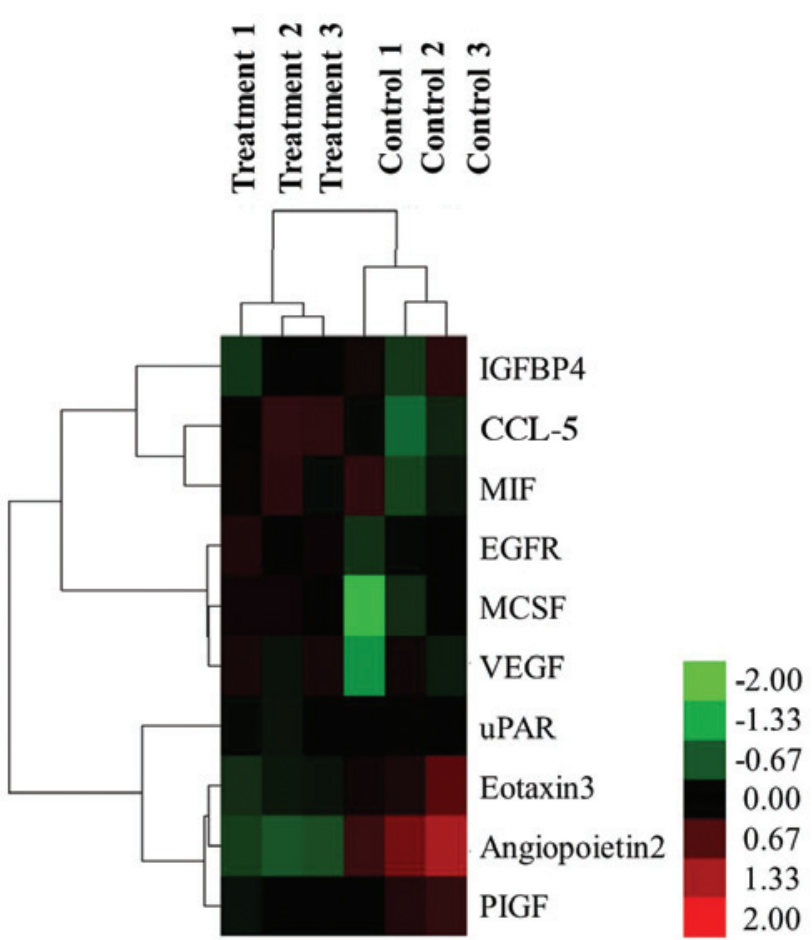

Figure 2. Hierarchical cluster analysis. Differentially secreted cytokines, which were defined as a $>1.5$-fold induction from control, were clustered according to their secretion (control or HMGB1-induced). Six HMGB1-induced cytokines (red) and four repressed cytokines (green) were detected. HMGB1, high mobility group box 1; IGFBP, insulin-like growth factor binding protein CCL-5, chemokine C-C motif ligand 5; MIF, macrophage migration inhibitory factor; EGFR, epidermal growth factor receptor; M-CSF, macrophage colony stimulating factor; VEGF, vascular endothelial growth factor; uPAR, urokinase plasminogen activator receptor; PIGF, placental growth factor.

were determined in the control and HMGB1-treated MSCs by western blotting. HMGB1 treatment induced a marked increase in the protein expression levels of GTP-Ras and this effect was sensitive to incubation with the Ras inhibitor. Notably, HMGB1 treatment increased the expression levels of p-p38 and p-ERK and this effect was sensitive to the incubation with the Ras inhibitor. These results suggests that HMGB1 activates the Ras signaling pathway and subsequently stimulates the p38 and ERK pathways of MSCs (Fig. 4A).

ALP staining and quantification of the expression of the late OCN was performed in MSCs. ALP activity and OCN gene expression levels were significantly higher in the HMGB1-stimulated MSCs, as compared with the control cells $(\mathrm{P}<0.01)$. Furthermore, as compared with the results in the HMGB1-induced group, the upregulation of ALP activity and OCN expression in MSCs was significantly reduced following Ras inhibitor treatment $(\mathrm{P}<0.01$; Figs. $4 \mathrm{~B}-\mathrm{D})$. These results suggest that HMGB1 potentiates the osteogenic differentiation of MSCs through the Ras/MAPK signaling pathway.

\section{Discussion}

Antibody array assays were performed in the present study to investigate cytokine secretion from HMGB1-stimulated MSCs, in order to elucidate the effects of HMGB1 on MSC functions. The results of the present study indicated that HMGB1 promotes
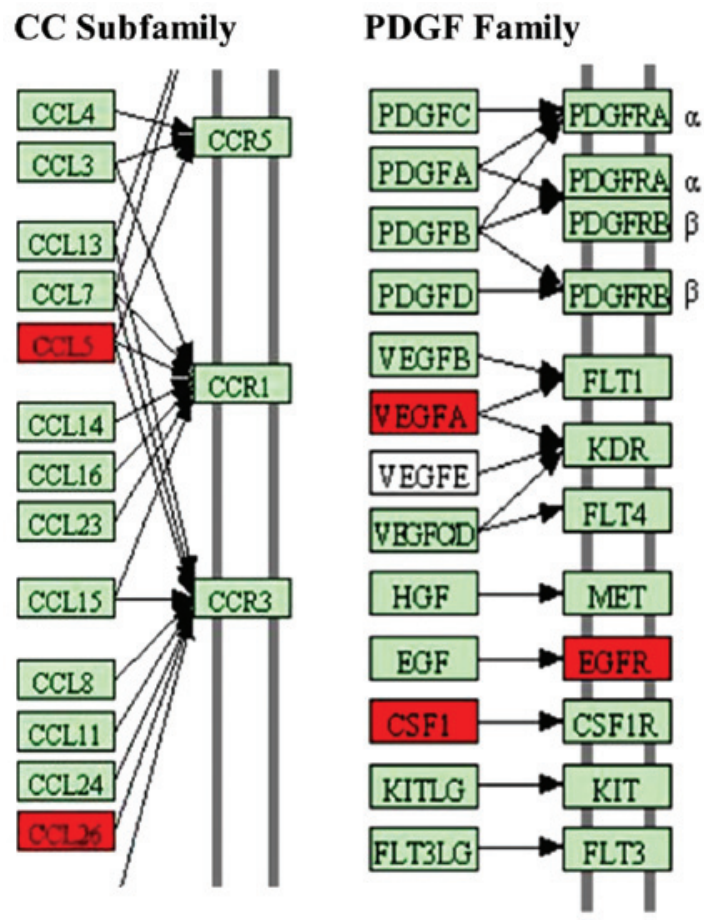

Figure 3. Cytokine-cytokine receptor interaction of secreted cytokines. CCL5, CCL26, VEGFA and CSF1 of the 10 differentially secreted cytokines involved cellular movement and cell proliferation processes were presented in their cytokine-cytokine receptor interaction pathway (CCR5, CCR1, CCR3, FLT1, KDR, EGFR and CSF1R) according to the cytokine-cytokine receptor interaction pathway (0460hsa) in Kyoto Encyclopedia of Genes and Genomes database. Red denotes an increase.

the secretion of various cytokines by MSCs. According to the Gene Ontology database for annotation, these secreted cytokines participate in various biological processes, including cell development, cell growth and migration, stress responses and immune system functions.

Among the cytokines secreted upon HMGB1 treatment in the present study, CCL5 and CCL26 are prominent chemocytokines that belong to the $\mathrm{CC}$ subfamily. The $\mathrm{CC}$ subfamily, which is also known as the $\beta$-chemokine subfamily, is the largest chemokine family, the members of which mainly act on monocytes and lymphocytes (22). In the fracture microenvironment, the secretion of CCL5 and CCL26 by HMGB1-stimulated MSCs may promote the migration of immune cells to tissues affected by the fracture sites, stimulating inflammatory responses and immune reactions (23-25). M-CSF, which is also known as human macrophage-specific colony-stimulating factor (CSF-1), is one of a few cytokines which are directly involved in osteoclastogenesis, osteoclast proliferation and differentiation $(26,27)$. VEGF is a specific heparin-binding growth factor in vascular endothelial cells, which can induce angiogenesis in vivo (28). It has previously been reported that VEGF could induce osteoclastogenesis (29), and the results of the present study indicate that VEGF and M-SCF are essential for the occurrence of osteoclastogenesis. During fracture restoration, the secretion of VEGF and M-SCF by MSCs was enhanced upon the HMGB1 treatment, leading to an increase in the cell number and activity osteoclasts. Although osteoclast cell activation will delay the coalescence of fractures during the early phase, it will facilitate reparative processes on the osteoblast surface during 
A

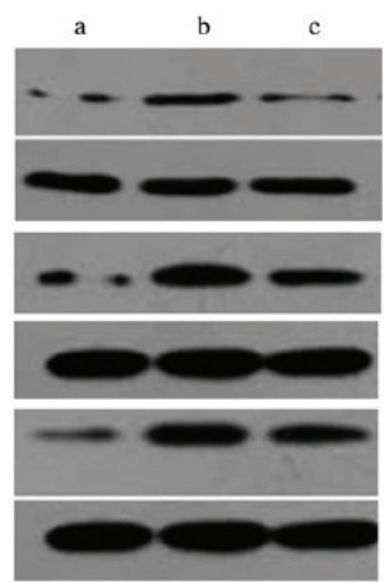

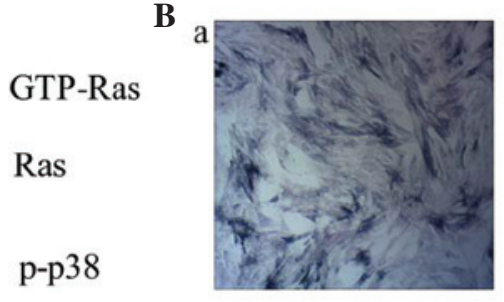

p38

p-ERK

ERK

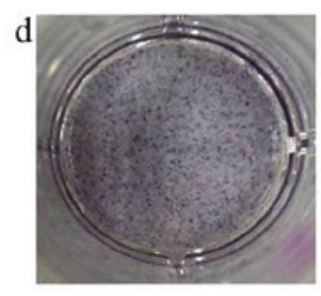

D
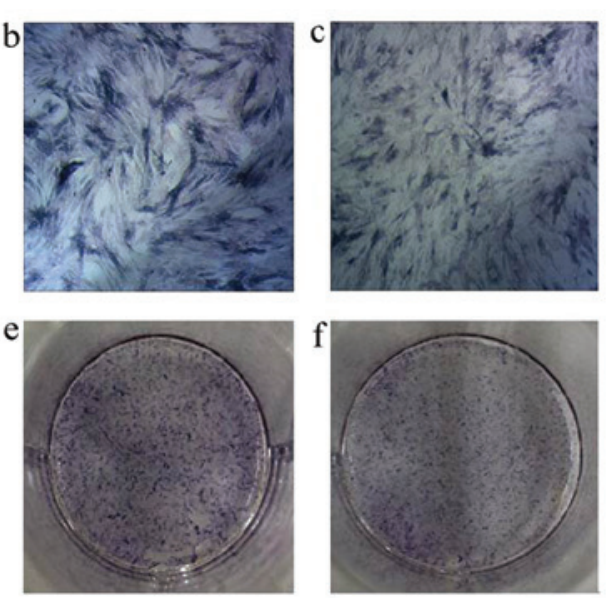

ALP activity

C

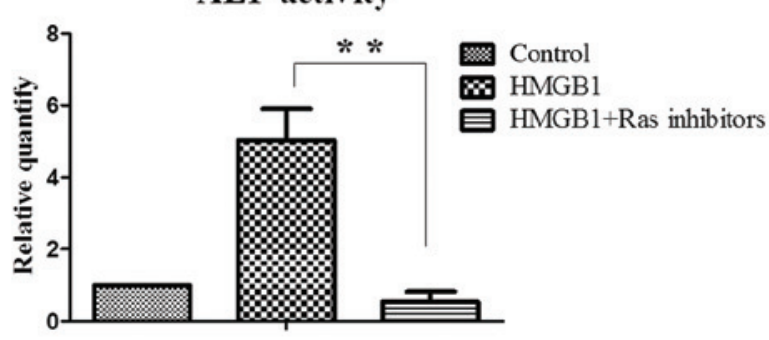

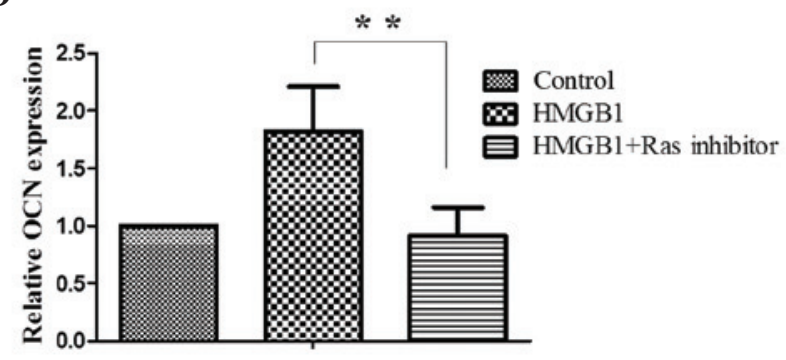

Figure 4. Ras activation is required for $\mathrm{p} 38$ and ERK activation and MSC osteogenesis. (A) Western blot analysis of Ras, p38, and ERK signaling activity was performed on (a) MSCs maintained in the basal medium alone, (b) with $25 \mathrm{ng} / \mathrm{ml} \mathrm{HMGB1}$ or (c) with $25 \mathrm{ng} / \mathrm{ml} \mathrm{HMGB1}$ and $5 \mu$ M Ras inhibitor. (B) On day 5 , ALP staining was performed on (a and d) MSCs maintained in the basal medium alone, (b and e) with $25 \mathrm{ng} / \mathrm{ml} \mathrm{HMGB1}$ or (c and f) with $25 \mathrm{ng} / \mathrm{ml} \mathrm{HMGB1}$ and $5 \mu \mathrm{M}$ Ras inhibitor. (C) Quantification of ALP activity among the groups. (D) Expression levels of the late osteogenesis marker OCN were determined by reverse transcription-quantitative polymerase chain reaction analysis in MSCs upon HMGB1 and/or Ras inhibitor treatment as indicated.* $\mathrm{P}<0.01$. HMGB1, high mobility group box 1; GTP, guanosine triphosphate; p-, phosphorylated; ERK, extracellular signal-regulated kinase; MSC, mesenchymal stem cell; ALP, alkaline phosphatase; OCN, osteocalcin.

the late phase (30,31). Therefore, further studies are necessary to investigate the effects of the HMGB1-promoted cytokine secretion by MSCs on osteoclast cells in detail. Such studies may provide a novel theoretical basis for improving fracture therapy.

Notably, EGFR expression levels were was markedly enhanced by HMGB1 stimulation in the present study. EGF is a polypeptide that belongs to the EGF superfamily and functions as a mutifunctional cytokine that can promote cell division and proliferation in vivo (32). The interaction between EGF and EGFR activates the Ras protein, which mediates the downstream signal transduction of various cytokines (33). Ras signaling regulates multiple downstream signaling pathways, which are predominantly implicated in cell proliferation and differentiation (34). It is widely accepted that osteogenesis depends on the activation of various key signaling pathways and that Ras/MAPK pathway regulates osteogenic differentiation of stem cells (35). Although the function of HMGB1 in osteogenic differentiation of MSCs has been established previously, the underlying mechanisms of HMGB1 effects remain unknown. Therefore, we hypothesize that HMGB1 may promote osteogenic differentiation of MSCs via the activation of Ras/MAPK signaling pathway in MSCs.

In the present study, it was demonstrated that the levels of GTP-bound Ras were increased upon HMGB1. It is known that only GTP-bound Ras interacts with Raf-1 kinase and stimulates the latter to initiate the MAPK signaling cascade (36). Because the level of GTP-bound Ras represents the activation of Ras (37), it is possible to conclude that Ras signaling activity may be activated by HMGB1 treatment. Similarly, the phosphorylation levels of p38 and ERK were upregulated in HMGB1-treated MSCs. These observations suggest that p38 and ERK signaling pathways were also activated as a result of Ras activation. An inhibitor of Ras was applied to block its activity in order to verify our hypothesis that p38 and ERK may be stimulated via the activated Ras signaling pathway. As hypothesized, the activation of ALP and the expression of OCN promoted by HMGB1 treatment were remarkably downregulated following Ras inhibitor treatment. These results demonstrated that the osteogenic differentiation of MSCs stimulated by HMGB1 is sensitive to Ras inhibition. Moreover, the upregulation of the expression levels of p-p38 and p-ERK upon treatment with HMGB1 were also reduced by the inhibition of Ras, suggesting that p38 and ERK pathways are suppressed when Ras activity is blocked.

On the basis of these observations, we conclude that HMGB1 activates the Ras signaling pathway and subsequently stimulates the p38 and ERK pathways to promote the osteogenic differentiation of MSCs. It is important to note that the activation of the Ras/MAPK signaling pathway is only one of various mechanisms affecting the HMGB1-induced osteogenic differentiation of MSCs, as numerous downstream signaling pathways are regulated by Ras signaling, including the Rap1 and PI3K-AKT cascades $(38,39)$. Therefore, the identity of the pathway(s) affected by the activation of Ras upon HMGB1 treatment and 
their roles in the osteogenic differentiation of MSCs should be investigated in future studies. In addition to exocrine cytokines, MSCs also synthesize endocrine proteins which regulate numerous biological processes (40), and it remains to be elucidated whether HMGB1 affects the endocrine protein synthesis. Therefore, future studies should focus on investigating differentially expressed endocrine proteins in HMGB1-stimulated MSCs to gain further insight into the mechanisms of regulation of MSC functions by HMGB1.

\section{Acknowledgements}

The present study was supported by a grant from the National Natural Science Foundation of China (grant nos. 81271973 and 81201397), the Zhejiang Provincial Natural Science Foundation of China (grant no. Y13H060003), the Department of Health Bureau of Zhejiang Province (grant no. 2014KYA092) and the Zhejiang Medical and Health Science and Technology Plan Project (grant no. 201462458).

\section{References}

1. Gardella S, Andrei C, Ferrera D, Lotti LV, Torrisi MR, Bianchi ME and Rubartelli A: The nuclear protein HMGB1 is secreted by monocytes via a non-classical, vesicle-mediated secretory pathway. EMBO Rep 3: 995-1001, 2002.

2. Fink MP: Bench-to-bedside review: High-mobility group box 1 and critical illness. Crit Care 11: 229, 2007.

3. Degryse B, Bonaldi T, Scaffidi P, Müller S, Resnati M, Sanvito F, Arrigoni G and Bianchi ME: The high mobility group (HMG) boxes of the nuclear protein HMG1 induce chemotaxis and cytoskeleton reorganization in rat smooth muscle cells. J Cell Biol 152: 1197-1206, 2001.

4. Palumbo R, Sampaolesi M, De Marchis F, Tonlorenzi R, Colombetti S, Mondino A, Cossu G and Bianchi ME: Extracellular HMGB1, a signal of tissue damage, induces mesoangioblast migration and proliferation. J Cell Biol 164: 441-449, 2004.

5. Andersson U, Erlandsson-Harris H, Yang H and Tracey KJ: HMGB1 as a DNA-binding cytokine. J Leukoc Biol 72: 1084-1091, 2002

6. Yang H, Wang H, Czura CJ and Tracey KJ: HMGB1 as a cytokine and therapeutic target. J Endotoxin Res 8: 469-472, 2002.

7. Naglova $\mathrm{H}$ and Bucova M: HMGB1 and its physiological and pathological roles. Bratisl Lek Listy 113: 163-171, 2012.

8. Granero-Moltó F, Weis JA, Miga MI, Landis B, Myers TJ, O'Rear L, Longobardi L, Jansen ED and Mortlock DP: Regenerative effects of transplanted mesenchymal stem cells in fracture healing. Stem Cells 27: 1887-1898, 2009.

9. Glass GE, Chan JK, Freidin A, Feldmann M, Horwood NJ and Nanchahal J: TNF-alpha promotes fracture repair by augmenting the recruitment and differentiation of muscle-derived stromal cells. Proc Natl Acad Sci USA 108: 1585-1590, 2011.

10. Marsell R and Einhorn TA: The biology of fracture healing. Injury 42: 551-555, 2011.

11. Mingari MC, Moretta A and Moretta L: Regulation of KIR expression in human T cells: A safety mechanism that may impair protective T-cell responses. Immunol Today 19: 153-157, 1998.

12. Guo Z, Yang J, Liu X, Li X, Hou C, Tang PH and Mao N: Biological features of mesenchymal stem cells from human bone marrow. Chin Med J (Engl) 114: 950-953, 2001.

13. Guo J, Jie W, Shen Z, Li M, Lan Y, Kong Y, Guo S, Li T and Zheng S: SCF increases cardiac stem cell migration through PI3K/AKT and MMP2/9 signaling. Int J Mol Med 34: 112-118, 2014.

14. Sidney LE, Kirkham GR and Buttery LD: Comparison of osteogenic differentiation of embryonic stem cells and primary osteoblasts revealed by responses to IL-1 $\beta$, TNF- $\alpha$ and IFN- $\gamma$. Stem Cells Dev 23: 605-617, 2014.

15. Tso GH, Law HK, Tu W, Chan GC and Lau YL: Phagocytosis of apoptotic cells modulates mesenchymal stem cells osteogenic differentiation to enhance IL-17 and RANKL expression on CD4+ T cells. Stem Cells 28: 939-954, 2010.
16. Meirelles Lda S, Fontes AM, Covas DT and Caplan AI: Mechanisms involved in the therapeutic properties of mesenchymal stem cells. Cytokine Growth Factor Rev 20: 419-427, 2009.

17. Yuk JM, Yang CS, Shin DM, Kim KK, Lee SK, Song YJ, Lee HM, Cho CH, Jeon BH and Jo EK: A dual regulatory role of apurinic/apyrimidinic endonuclease 1/redox factor- 1 in HMGB1-induced inflammatory responses. Antioxid Redox Signal 11: 575-588, 2009.

18. Lee W, Ku SK, Bae JW and Bae JS: Inhibitory effects of lycopene on HMGB1-mediated pro-inflammatory responses in both cellular and animal models. Food Chem Toxicol 50: 1826-1833, 2012.

19. Meng E, Guo Z, Wang H, Jin J, Wang J, Wang H, Wu C and Wang L: High mobility group box 1 protein inhibits the proliferation of human mesenchymal stem cells and promotes their migration and differentiation along osteoblastic pathway. Stem Cells Dev 17: 805-813, 2008.

20. Livak KJ and Schmittgen TD: Analysis of relative gene expression data using real-time quantitative PCR and the $2^{-\Delta \Delta C}$ method. Methods 25: 402-408, 2001.

21. Nonomura A, Ohta G, Nakanuma Y, Izumi R, Mizukami Y, Matsubara F, Hayashi M, Watanabe K and Takayanagi N: Simultaneous detection of epidermal growth factor receptor (EGF-R), epidermal growth factor (EGF) and ras p21 in cholangiocarcinoma by an immunocytochemical method. Liver 8: 157-166, 1988.

22. Zlotnik A and Yoshie O: Chemokines: A new classification system and their role in immunity. Immunity $12: 121-127$, 2000.

23. Pham K, Luo D, Liu C and Harrison JK: CCL5, CCR1 and CCR5 in murine glioblastoma: Immune cell infiltration and survival rates are not dependent on individual expression of either CCR1 or CCR5. J Neuroimmunol 246: 10-17, 2012.

24. Lee JK, Schuchman EH, Jin HK and Bae JS: Soluble CCL5 derived from bone marrow-derived mesenchymal stem cells and activated by amyloid $\beta$ ameliorates Alzheimer's disease in mice by recruiting bone marrow-induced microglia immune responses. Stem Cells 30: 1544-1555, 2012.

25. Errahali YJ, Taka E, Abonyo BO and Heiman AS: CCL26-targeted siRNA treatment of alveolar type II cells decreases expression of CCR3-binding chemokines and reduces eosinophil migration: Implications in asthma therapy. J Interferon Cytokine Res 29: 227-239, 2009.

26. De Vries TJ, Schoenmaker T, Aerts D, Grevers LC, Souza PP, Nazmi K, van de Wiel M, Ylstra B, Lent PL, Leenen PJ and Everts V: M-CSF priming of osteoclast precursors can cause osteoclastogenesis-insensitivity, which can be prevented and overcome on bone. J Cell Physiol 230: 210-225, 2015.

27. Karst M, Gorny G, Galvin RJ and Oursler MJ: Roles of stromal cell RANKL, OPG and M-CSF expression in biphasic TGF-beta regulation of osteoclast differentiation. J Cell Physiol 200: 99-106, 2004.

28. Kazemi-Lomedasht F, Behdani M, Bagheri KP, Habibi-Anbouhi M, Abolhassani M, Arezumand R, Shahbazzadeh D and Mirzahoseini H: Inhibition of angiogenesis in human endothelial cell using VEGF specific nanobody. Mol Immunol 65: 58-67, 2015.

29. Henriksen K, Karsdal M, Delaisse JM and Engsig MT: RANKL and vascular endothelial growth factor (VEGF) induce osteoclast chemotaxis through an ERK1/2-dependent mechanism. J Biol Chem 278: 48745-48753, 2003.

30. Kayal RA, Tsatsas D, Bauer MA, Allen B, Al-Sebaei MO, Kakar S, Leone CW, Morgan EF, Gerstenfeld LC, Einhorn TA and Graves DT: Diminished bone formation during diabetic fracture healing is related to the premature resorption of cartilage associated with increased osteoclast activity. J Bone Miner Res 22: 560-568, 2007.

31. Lee K, Kim H, Kim JM, Kim JR, Kim KJ, Kim YJ, Park SI, Jeong JH, Moon YM, Lim HS, et al: Systemic transplantation of human adipose-derived stem cells stimulates bone repair by promoting osteoblast and osteoclast function. J Cell Mol Med 15: 2082-2094, 2011.

32. Wang CL, Jiang H and Sun JB: EGF and SCF promote the proliferation and differentiation of mouse spermatogenic cells in vitro. Zhonghua Nan Ke Xue 20: 679-683, 2014 (In Chinese).

33. Kikuchi A, Amagai M, Hayakawa K, Ueda M, Hirohashi S, Shimizu N and Nishikawa T: Association of EGF receptor expression with proliferating cells and of ras p21 expression with differentiating cells in various skin tumours. Br J Dermatol 123: 49-58, 1990. 
34. Scholz ME, Meissner JD, Scheibe RJ, Umeda PK, Chang KC, Gros G and Kubis HP: Different roles of H-ras for regulation of myosin heavy chain promoters in satellite cell-derived muscle cell culture during proliferation and differentiation. Am J Physiol Cel Physiol 297: C1012-C1018, 2009.

35. Peng S, Zhou G, Luk KD, Cheung KM, Li Z, Lam WM, Zhou Z and Lu WW: Strontium promotes osteogenic differentiation of mesenchymal stem cells through the Ras/MAPK signaling pathway. Cell Physiol Biochem 23: 165-174, 2009.

36. Yao B, Zhang Y, Delikat S, Mathias S, Basu S and Kolesnick R: Phosphorylation of Raf by ceramide-activated protein kinase. Nature 378: 307-310, 1995.

37. Mor A and Philips MR: Compartmentalized Ras/MAPK signaling. Annu Rev Immunol 24: 771-800, 2006.
38. Zeiller C, Blanchard MP,Pertuit M, Thirion S, Enjalbert A, Barlier A and Gerard C: Ras and Rap1 govern spatiotemporal dynamic of activated ERK in pituitary living cells. Cell Signal 24: 2237-2248, 2012.

39. Hubbard PA, Moody CL and Murali R: Allosteric modulation of Ras and the PI3K/AKT/mTOR pathway: Emerging therapeutic opportunities. Front Physiol 5: 478, 2014.

40. Binger T, Stich S, Andreas K, Kaps C, Sezer O, Notter M, Sittinger $M$ and Ringe J: Migration potential and gene expression profile of human mesenchymal stem cells induced by CCL25. Exp Cell Res 315: 1468-1479, 2009 